\title{
DIFFUSIVE SYSTEMS AND WEIGHTED HANKEL OPERATORS
}

\author{
Aolo Bashar Abusaksaka And Jonathan R. Partington
}

Abstract. We consider diffusive systems, regarded as input/output systems with a kernel given as the Fourier-Borel transform of a measure in the left half-plane. Associated with these are a family of weighted Hankel integral operators, and we provide conditions for them to be bounded, Hilbert-Schmidt or nuclear, thereby generalizing results of Widom, Howland and others.

Mathematics subject classification (2010): 47B35, 46E15, 35K05, 93B28.

Keywords and phrases: Hankel operator, nuclear operator, Carleson embedding, Laplace transform, diffusive system, heat equation, Fourier-Borel transform.

\section{REFERENCES}

[1] A. Bashar Abus aks aka, Properties of delay systems and diffusive systems, Ph. D. thesis, University of Leeds (2015).

[2] F. F. Bons ALL, Boundedness of Hankel matrices, J. London Math. Soc. (2) 29 (1984), no. 2, 289-300.

[3] C. Casenave And G. Montseny, Introduction to diffusive representation, 4th IFAC syposium on system, structure and control, (2010), 15-17. https://hal.archives-ouvertes.fr/hal $-00854381$.

[4] E. B. DAVIES, Linear operators and their spectra, Cambridge Studies in Advanced Mathematics 106, Cambridge University Press, Cambridge, 2007.

[5] K. Glover, R. F. Curtain And J. R. Partington, Realisation and approximation of linear infinite-dimensional systems with error bounds, SIAM J. Control Optim. 26 (1988), no. 4, 863-898.

[6] K. Glover, J. Lam And J. R. Partington, Rational approximation of a class of infinitedimensional systems: the $L_{2}$ case, Progress in approximation theory, 405-440, Academic Press, Boston, MA, 1991.

[7] C. GUIVER AND M. R. OPMEeR, Model reduction by balanced truncation for systems with nuclear Hankel operators, SIAM J. Control Optim. 52 (2014), no. 2, 1366-1401.

[8] J. S. Howland, Trace class Hankel operators, Quart. J. Math. Oxford Ser. (2) 221971 147-159.

[9] B. Jacob, J. R. Partington And S. Pott, On Laplace-Carleson embedding theorems, J. Funct. Anal. 264 (2013), no. 3, 783-814.

[10] B. JaCOB, J. R. Partington And S. Pott, Applications of Laplace-Carleson embeddings to admissibility and controllability, SIAM J. Control Optim. 52 (2014), no. 2, 1299-1313.

[11] G. MonTSENy, Diffusive representation of pseudo-differential time-operators, ESAIM Proceedings 5 (1998), 159-175.

[12] G. Montseny, Représentation diffusive, Lavoisier Hermes-Science, Paris (2005).

[13] S. C. Power, Hankel operators on Hilbert space, Research Notes in Mathematics 64, Pitman (Advanced Publishing Program), Boston, Mass., London, 1982.

[14] H. Widom, Hankel matrices, Trans. Amer. Math. Soc. 121 (1966), 1-35. 